\title{
APROXIMACIÓN HISTÓRICA Y METODOLÓGICA A LOS CURSOS DE PEDAGOGÍA MUSICAL DE LA UNIVERSITAT DE LES ILLES BALEARS (1977-1990)
}

\section{Historical and methodological approach to music education courses at the Universitat de les Illes Balears (1977-I990)}

Llorenç Gelabert GuAL ${ }^{1}$

Departamento de Pedagogía y Didácticas Específicas. Universitat de les Illes Balears

Correo-e: llorens.gelabert@uib.cat

Recepción: 30 de abril de 20I4. Envío a informantes: Is de mayo de 2014.

Fecha de aceptación definitiva: 27 de junio de 2014

Resumen: En la inercia del incipiente paradigma educativo gestado en España en la década de los setenta del siglo pasado, un grupo de músicos y profesores llevaron a cabo una iniciativa formativa con la intención de introducir la música en las escuelas de las Islas Baleares. Enmarcados en el ámbito no formal, estos cursos se convirtieron en el hilo transmisor de una nueva concepción pedagógica. Dicha concepción se basaba en las metodologías activas de vanguardia aplicadas con éxito en otros países de Europa y el especial vínculo de trabajo establecido con el entorno pedagógico del músico catalán Ireneu Segarra. Este artículo pretende aproximarnos al legado de esta oferta formativa desde las vertientes histórica y metodológica, remarcando también la potencialidad y utilidad de un formato de cursos similar a día de hoy, una vez desaparecida la especialidad del Maestro en Educación Musical en el nuevo marco de adaptación al Espacio Europeo de Educación Superior.

Palabras Clave: reformas e innovaciones educativas; Renovación Educativa; Pedagogía musical; Didáctica musical; Historia de la Educación; siglo xx.

- El autor de este texto es miembro del Grup d'Estudis d'Història de l'Educació de la Universitat de les Illes Balears, que ha recibido el patrocinio de la Comunidad Autónoma de las Illes Balears y más concretamente de la Direcció General de Recerca, Desenvolupament Tecnològic i Innovació de la Conselleria d'Innovació, Interior i Justícia; y el cofinanciamiento con fondos FEDER. 
APROXIMACIÓN HISTÓRICA Y METODOLÓGICA A LOS CURSOS DE PEDAGOGÍA MUSICAL DE LA UNIVERSITAT DE LES ILLES BALEARS (I977-I990) LLORENÇ GELABERT GUAL

AвSTRACT: The education in general and the music in particular were not outside to the changes developed in Spain in the seventies of last century. The initiative, in the form of summer courses, promoted by a group of teachers and musicians, will mean the beginning of a true outcrop in music education, and will get in crescendo. This new concept is based on active methodologies successfully applied in other European countries and their linkage with the learning environment of the Catalan musician Ireneu Segarra, in the line of great figures of the musical avantgarde. This article is intended to approach the legacy of these courses from two perspectives. First, from a historical point of view, and then reaching any specific methodological applications then initiated. Finally, this article intends to approach his legacy highlighting the potential and utility also could acquire a similar format nowadays, in the new adaptation framework to EHEA.

KEY wORDs: Educational innovations and reforms; Educational renewal; Music Education; Music Teachers; History of Education; $20^{\mathrm{TH}}$ century.

\section{Introducción}

$\mathrm{L}$

A TRAYECTORIA Y LEGADO DE LOS CURSOS DE PEDAGOGÍA MUSICAL, objeto de análisis en este artículo, nacen del originario I Curs de Música Coral organizado por la Cátedra de Música de la Escuela de Formación del Profesorado de Palma en el verano de i977. Pese a enmarcarse en el ámbito de la educación no formal, la organización debió su tutela y amparo legal a la mencionada institución en los primeros años y posteriormente a la Universitat de les Illes Balears. En la medida en que se fueron añadiendo nuevas especialidades y disciplinas, los cursos acabaron asumiendo un rol formativo que las instituciones educativas de entonces no contemplaban. Aunque gran parte del peso lectivo recayó inicialmente en la formación en dirección coral, la demanda de recursos para el aula de música por parte de un numeroso colectivo de maestros propició la creación y rápida consolidación de la especialidad de Pedagogía Musical. Dicho auge se entendía como una consecuencia de la inacción en cuanto a formación del profesorado en música durante la etapa franquista ${ }^{2}$. El declive de la especialidad, trece años después, coincidió con la creación en las universidades españolas de los estudios de Maestro en Educación Musical, en el marco de la Ley Orgánica General del Sistema Educativo (LOGSE) de 1990. El reconocimiento de esta nueva disciplina universitaria suplió y formalizó el espacio que hasta entonces habían ocupado los cursos ${ }^{3}$.

En el marco vigente de adaptación de los estudios universitarios al Espacio Europeo de Educación Superior (EEES), desaparecen las especialidades de maestro que introdujo la LOGSE a favor de las figuras generalistas que contemplan los

En cuanto a la música en las escuelas durante el franquismo y el profesorado encargado de impartirla v. CASTAÑón, M. R.: «El profesorado de educación musical durante el franquismo», REIFOP (Revista Interuniversitaria de Formación del Profesorado), I2 (2009), pp. 97-I07.

3 DíAz, B. M.: «La Educación Musical en la Escuela y el Espacio Europeo de Educación Superior», Revista Interuniversitaria de Formación del Profesorado, 52 (2005), pp. 23-38. 
APROXIMACIÓN HISTÓRICA Y METODOLÓGICA A LOS CURSOS DE PEDAGOGÍA MUSICAL DE LA UNIVERSITAT DE LES ILLES BALEARS (I977-I990) LLORENÇ GELABERT GUAL

estudios de Grado en Educación Infantil y Primaria ${ }^{4}$. Este nuevo contexto podría dar sentido de nuevo a una oferta formativa similar al formato de cursos objeto de este estudio, al desaparecer la formación específica en pedagogía y didáctica musical, tal y como ocurrió en la década de los setenta del siglo pasado.

Con el objetivo de profundizar en esta propuesta formativa analizaremos el eco social y el legado metodológico que aportaron los cursos, partiendo de un análisis del contexto histórico y legislativo en que tuvieron lugar. Esta reflexión inicial pretende responder a la pregunta: ¿de dónde venimos? El devenir de los mismos será también objeto de comentario y vendrá establecido por el grado de regulación y reconocimiento de las enseñanzas musicales en los diferentes niveles e instituciones de enseñanza.

Baltasar Bibiloni', compositor y profesor en el área de Didáctica de la Expresión Musical de la Universitat de les Illes Balears, y Joan Company ${ }^{6}$, director y fundador de la Coral Universitat de les Illes Balears, fueron los creadores e impulsores de la iniciativa ${ }^{7}$. No es coincidencia que derivaran además, ya desde los inicios, en la conjunción del ámbito de la música coral con la pedagogía musical, espacios íntimamente conexos por el canto común y la técnica vocal. Grandes pedagogos europeos del siglo xx ya sitúan como eje de la enseñanza musical en la escuela a dicha faceta musical. Bibiloni y Company se sumarán también a esta idea desde su propuesta formativa.

4 Sobre el reconocimiento de las enseñanzas musicales en el nuevo marco del Espacio Europeo de Educación Superior (eEes) v. Díaz Mohedo, M. T.: «La Educación Musical en la Europa del futuro», Música y Educación: Revista Trimestral de Pedagogía Musical, 68 (2006), pp. 34-48.

Baltasar Bibiloni i Llabrés (1936-), compositor y pedagogo musical. Fue profesor en la Escuela de Formación del Profesorado de EGB de Palma y posteriormente en la Universitat de les Illes Balears, en el área de Didáctica de la Expresión Musical. Trabajó también en el Departamento de Pedagogía Musical del Conservatori Superior de Música i Dansa de les Illes Balears. Como compositor destacan sus arreglos de música tradicional de las Islas Baleares adaptados a la escuela primaria: Cinc cançons mallorquines (1980) o 20 cançons tradicionals per a veus blanques i piano (1996), etc.; y sus composiciones a partir de textos de autores en lengua catalana: Set poemes de Llorenç Moyà (1997), Cantata de la Fosca $i$ de la Llum (1999), Transatlàntida (200I), entre otros. Como director de numerosas agrupaciones corales destaca su etapa al frente de la Escolanía de Lluc (1993-200I), una de las agrupaciones de ese estilo más longevas de Europa. Ha recibido premios y distinciones por parte de instituciones como el Ajuntament de Binissalem, la Universitat de les Illes Balears, la Federació de Corals de les Illes Balears o l'Obra Cultural Balear. En cuanto a la aportación pedagógica de Baltasar Bibiloni v. Gelabert, Ll. y Miranda, J.: «Baltasar Bibiloni, un pedagogo musical», Música y Educación: Revista Trimestral de Pedagogía Musical, 92 (2012), pp. 36-49.

6 Joan Company i Florit (1954-), es director y fundador de la Coral Universitat de les Illes Balears (1977). Ha trabajado, entre otros, con el Orfeón Donostiarra, Coro Nacional de España, Coro de Radio Televisión Española, Coro de la Comunidad de Madrid. Ha estrenado obras de J. Busto, A. Parera Fons, J. Vila o J. L. Turina. Ha colaborado con directores como Trevor Pinnock, Víctor Pablo, Salvador Mas, Edmon Colomer, Josep Pons, Franz P. Decker, Alberto Zedda, A. Ros Marbà, C. Hogwood o Jesús López Cobos. Desde el año 1999 es director artístico del Coro de la Orquesta Sinfónica de Galicia. Ha publicado numerosos estudios y artículos sobre música y músicos de las Islas Baleares en libros, enciclopedias y revistas especializadas. Ha recibido, entre otras distinciones, el Premi Rotary Mallorca de Humanidades $1995-96$ y el Premi Gabriel Alomar $200 \mathrm{I}$ de l'Obra Cultural Balear.

Gelabert, Ll.: «Dues iniciatives en l'àmbit no formal de l'ensenyament musical a Mallorca (1977-1995)», Educació i Història. Revista d'Història de l'Educació, 20 (2012), pp. I4I-I60. 
APROXIMACIÓN HISTÓRICA Y METODOLÓGICA A LOS CURSOS DE PEDAGOGÍA MUSICAL DE LA UNIVERSITAT DE LES ILLES BALEARS (I977-I990) LLORENÇ GELABERT GUAL

\section{La enseñanza musical en España}

La música irá aumentando su peso lectivo dentro de los diferentes modelos y sistemas educativos que se van configurando en España a lo largo de los últimos ciento cincuenta años. Sin embargo, en el ámbito de las escuelas de enseñanza primaria, nunca aparece como materia o asignatura propia y definida como tal, y permanece en ese estatus hoy en día, siempre englobada en el área de Educación Artística. En un estudio elaborado por Inmaculada Egido sobre la evolución de la enseñanza primaria en España podemos visualizar la presencia de la música en los distintos contextos legislativos hasta la implantación de la LOGSE de $1990^{8}$.

Si bien los conservatorios son las instituciones encargadas de la formación específicamente musical proyectada al ámbito profesional, la tarea del maestro se enmarca y debe contribuir a la formación y educación integral del alumno a través de la música. Dicha formación integral debe principiarse ineludiblemente a partir de un contacto o sensibilización básica en la etapa de Educación Infantil, tal y como reconocen Akoschky, Alsina, Díaz y Giráldez ${ }^{9}$ y ampliarse a lo largo de la etapa de Educación Primaria. Baltasar Bibiloni, impulsor de los cursos objeto de este estudio y activista incesante a favor de la presencia de la música en la escuela, argumenta: «El conservatorio forma a los profesionales que un día ocuparán los escenarios musicales. Los maestros de educación musical actuarán, desde la escuela, a favor de que sus alumnos, el día de mañana, se conviertan en oyentes lúcidos que llenen las salas de conciertos» ${ }^{10}$.

Esta última competencia atribuida a la escuela es corroborada también por figuras relevantes de la historia de la pedagogía. La realidad, sin embargo, nos demuestra que la introducción de la enseñanza de la música en las escuelas españolas ha sido muy lenta y tardía. En el artículo 3 del Real Decreto de 26 de octubre de I90I, aprobado por el Ministerio de Instrucción Pública, encontramos una primera referencia a la presencia de la música entre las materias de primera enseñanza ${ }^{\text {II }}$. No consta, en cambio, ninguna referencia a la forma y perfil del maestro encargado de impartirla. Durante el periodo franquista no se visualiza ningún avance al respecto. Existen cambios relevantes en esa época en tanto en cuanto quedará diferenciada la educación entre ambos sexos, tanto en ubicación como en contenidos, dejando patente así la afinidad del régimen con los principios pedagógicos del catolicismo más reaccionario de épocas pretéritas. Entre las materias de la Sección Femenina se contemplaba la formación musical en solfeo y canto, basada en una formación inicial y reducida al aprendizaje del lenguaje musical y un repertorio básico de canciones $^{12}$.

8 Egido Gálvez, I.: «La evolución de la enseñanza primaria en España: organización de la etapa y programas de estudio», Revista Tendencias Pedagógicas, I (1995), pp. 75-86.

9 Aкоochky, J.; Alsina, P.; Díaz, M. y Giráldez, A.: La música en la escuela infantil (o-6), Barcelona, Editorial Graó, 2008.

10 Bibiloni, B.: «Música i estudis de mestre», Estudis Baleàrics, 80/8I (2006), pp. I29-I33.

II Real Decreto de 26 de octubre de I90I, artículo 3. Aparece el listado de materias de primera enseñanza pública. El canto es la novena materia en orden de aparición.

12 Candela, M. A. y Montero, J.: Música. Teoría de solfeo y canciones, Sección Femenina de FET y de las JONs (quinta edición), 1959. 
APROXIMACIÓN HISTÓRICA Y METODOLÓGICA A LOS CURSOS DE PEDAGOGÍA MUSICAL DE LA UNIVERSITAT DE LES ILLES BALEARS (I977-I990) LLORENÇ GELABERT GUAL

Con la aprobación de la Ley General de Educación (LGE) de 1970, la expresión musical, como bloque incluido en el área de Educación artística, ya tiene cabida en el nuevo plan de estudios ${ }^{13}$. Hablamos de una inédita secuenciación de contenidos que en cierto modo debe contribuir a la consolidación y normalización de la enseñanza musical en las escuelas. Nos preguntamos entonces: ¿Se llevó a la práctica dicha implantación y normalización? La respuesta taxativa es no. El principal motivo se debía a la falta de profesorado formado en pedagogía musical aplicada a la escuela primaria ${ }^{14}$. Precisamente en ese contexto nacieron los cursos objeto de este estudio, asumiendo el rol formativo que las escuelas de maestros, en cuanto a música, no contemplaban. Enmarcados en el ámbito no formal pretenden, desde sus inicios, aportar formación y recursos en pedagogía y didáctica musicales aplicadas a la escuela. En cuanto a la difusión y aplicación de metodologías activas, tanto en música como en otras disciplinas presentes en la escuela, los cursos de la Universitat de les Illes Balears se adhirieron a una corriente de propuestas formativas ya implantada entonces en Cataluña y que gozaba de gran prestigio y repercusión. En esta línea destacan instituciones como la escuela de maestros Rosa Sensat o la Escola de Pedagogia Musical-Mètode Irenen Segarra.

La LOGSE de 1990, en las llamadas Enseñanzas de Régimen General, contemplaba, respecto a leyes precedentes, la mayor cota de reconocimiento de las enseñanzas artísticas y por ende musicales. Ya en la etapa de Educación Infantil se incluye la iniciación a la sensibilización y vivencia musical a través del área de Comunicación y Representación. Se reconoce en el nuevo texto, por primera vez y de forma explícita, la importancia de la educación musical en esta etapa educativa $^{\text {Is. }}$. En la etapa de Educación Primaria la música aparece integrada de nuevo en el área de Educación Artística. Los bloques temáticos incluyen: el canto, la expresión vocal e instrumental y el lenguaje musical, que abarca la formación rítmica, el movimiento y la danza, la lectura y la audición. Sin embargo, y más allá del ambicioso plan de estudios, sólo dos de los bloques temáticos que abarcan la Educación artística en la Educación Primaria serán propiamente musicales. Estos dos bloques son: Canto, expresión vocal e instrumental y Lenguaje musical ${ }^{16}$.

La introducción de la especialidad de Maestro en Educación Musical en los estudios universitarios será una de las mayores aportaciones por parte de la LOGSE en cuanto a enseñanza musical. Paradójicamente, dicha introducción no conlleva reconocimiento del ámbito de la expresión musical como área curricular propia, que permanecerá en el mismo estatus, asociada al área de Educación Artística ${ }^{17}$.

13 El artículo i6 de la Ley I4/1970 de 4 de agosto, General de Educación y Financiación de la Reforma Educativa, refleja la iniciación en la apreciación y expresión estética y artística. Los contenidos en materia de educación musical, incluidos en el área de Educación Artística, vienen detallados en: Orden de 17 de enero de $198 \mathrm{I}$ ( $B O E$ núm. I8 de oI/2I/I98I), por la que se regulan las Enseñanzas de Educación Preescolar y del ciclo inicial de la Educación General Básica.

${ }_{14}$ Díaz, B. M.: op. cit. (2).

is Akoschky, J.; Alsina, P.; Díaz, M. y Giráldez, A.: op. cit. (8).

${ }_{16}$ Real Decreto I006/199I, de I4 de junio (BOE núm. I52 de 26/6/199i). En este se establecen los objetivos correspondientes a la etapa de Educación Primaria en todas las áreas de enseñanza. Entre ellas la Educación Artística que incluye, además de la expresión musical, la expresión plástica y la dramatización.

${ }^{17}$ Bibiloni, B.: op. cit. (9). 
APROXIMACIÓN HISTÓRICA Y METODOLÓGICA A LOS CURSOS DE PEDAGOGÍA MUSICAL DE LA UNIVERSITAT DE LES ILLES BALEARS (I977-I990)

LLORENÇ GELABERT GUAL

La LOE, ley actualmente en vigor y en la línea de la anterior, se ha aplicado no exenta de ciertas polémicas vinculadas a la presencia lectiva de la enseñanza musical en la etapa de primaria y secundaria. Dicha presencia se ve mermada en ciertas comunidades autónomas.

La valoración final respecto a la evolución de la enseñanza musical en España y en las Islas Baleares, a lo largo del último siglo, podemos calificarla objetivamente de positiva. Actualmente la formación musical se contempla ya en la etapa de Educación Infantil, Primaria y Secundaria. Existen también los conservatorios de grado elemental, medio y superior. Además, muchos municipios españoles cuen$\tan$ ya con escuelas de música municipales que ofrecen la posibilidad de una formación reglada.

\section{Instituciones de enseñanza musical en Mallorca}

En el año I879 nace la Sociedad Musical, que funcionará como Conservatorio Balear a partir de i88I, bajo la tutela del Ayuntamiento de Palma. En cuanto a instituciones de enseñanza musical sólo la preceden la longeva Escolanía de Lluc, de principios del siglo xvi, y la capilla de la Catedral de Mallorca. El Conservatorio Balear se disuelve en el año i886 por razones de inviabilidad económica. Cabe destacar la actividad formativa llevada a cabo por las bandas de música a lo largo de la segunda mitad del siglo XIX ${ }^{18}$.

El que es hoy el Conservatorio Profesional de Música y Danza de Mallorca se fundó en el año 1935 como Conservatorio Regional de Baleares mediante la Orden Ministerial de I9 de septiembre de 1935 del Ministerio de Instrucción Pública y Bellas Artes, siendo el primer director D. Nicolás Brondo. Destaca en los inicios la calidad pedagógica y artística de su profesorado. Esta brillante etapa se vio truncada de nuevo por una resolución de la Dirección General de Bellas Artes del año 1942, donde se ve rebajada la categoría del conservatorio profesional a elemental. En el año 1977 pasa de nuevo a profesional, en el marco de la ley de educación de 1970. La creación del Conservatorio Superior de Música y Danza de las Islas Baleares en el año 20or significa el paso definitivo a favor del reconocimiento de los estudios musicales como titulación superior; será la culminación de más de cien años de demanda de formación musical enfocada al ámbito profesional en las Islas Baleares ${ }^{19}$.

La Escuela Normal de maestros de Baleares, fundada en el año I842, fue el primer centro de formación de maestros de Mallorca. Entonces no se contemplaba la formación musical en los planes de estudios. Fue a partir del año i9i4 cuando se convierte en materia obligada en la formación de todos los maestros.

18 Sobre agrupaciones instrumentales y corales, asociacionismo y educación musical en Mallorca en el siglo xix v. Thomès, J. M. y Parets, J.: Breu Història Musical de les Illes Balears, Palma de Mallorca, Consell de Mallorca, Col-lecció Música I, Centre de Recerca i Documentació Historico Musical de Mallorca, 1989.

19 En cuanto a la historia del Conservatorio Balear y del actual Conservatorio Profesional de Música y Danza de Mallorca v. Roig, J.: «Conservatori, 70 anys d'ensenyament musical, I935-2005», Revista Estudis Baleàrics, 80/8I (2006), pp. I29-I33. 
APROXIMACIÓN HISTÓRICA Y METODOLÓGICA A LOS CURSOS DE PEDAGOGÍA MUSICAL DE LA UNIVERSITAT DE LES ILLES BALEARS (I977-I990)

LLORENÇ GELABERT GUAL

Los contenidos se basaban en la lectura musical o solfeo y en el aprendizaje de un repertorio básico de canciones. Hasta el año 1936 se ofrecía incluso la posibilidad de iniciarse en el estudio del piano. A partir del año i97I la música perderá espacio lectivo en todas las especialidades (Ciencias Sociales, Filología y Ciencias), salvo en la de Preescolar. En el caso de la Escuela Normal de Palma se pudo incluir una asignatura optativa de música poco tiempo después ${ }^{20}$.

El nuevo plan de estudios que contempla la especialidad de Maestro en Educación Musical, en el marco de la LOGSE, incluye materias que abarcan la formación en el lenguaje musical, formación instrumental, didáctica e historia de la música. La Universitat de les Illes Balears acogió esta nueva especialidad en el curso I990-9I y fue una de las primeras universidades de España en implantarla. Sin embargo, en el curso 2009-IO, y coincidiendo con la adaptación de los estudios universitarios al EEes que contempla la Ley Orgánica 6/200I de Universidades (LOU), desaparece la especialidad de Maestro en Educación Musical. De las siete especialidades que contempla la LOGSE pasamos a dos de carácter más generalista, el Grado en Educación Infantil y Primaria. La segunda incluirá unos pequeños itinerarios específicos de menor peso lectivo llamados menciones, que vienen a sustituir a las antiguas especialidades. La Mención de Música en los estudios de Grado en Educación Primaria en la Universitat de les Illes Balears incluye un total de treinta créditos ECTs. Doce de ellos son de carácter obligatorio para todos los estudiantes del Grado, y los restantes incluyen materias como la didáctica musical, la educación de la voz, agrupaciones instrumentales y vocales, y un periodo de prácticas específico. En el caso de los estudios de Grado en Educación Infantil se dedican doce créditos a la formación y sensibilización musical ${ }^{21}$.

Cabe destacar la labor desempeñada, en el ámbito no formal, por las escuelas de música municipales desde finales de la década de los noventa del siglo pasado. La demanda creciente de formación musical, únicamente en manos de los conservatorios o recluida al ámbito privado hasta entonces, dio paso a la creación de escuelas tuteladas por los ayuntamientos. Ello facilitó el acceso a la formación musical a un espectro más amplio de población. Con la LOGSE de I990 se dio un paso más en cuanto al reconocimiento de dichos centros y los estudios que en ellos se impartían. Hoy en día constituyen un pilar formativo de gran importancia que nutre de alumnos mejor preparados a los conservatorios profesional y superior ${ }^{22}$.

20 Sobre la evolución de la Escuela Normal de maestros de Palma y el magisterio en las Islas Baleares v. SuREdA, B.: La formación del profesorado en Mallorca. Antecedentes y origen de la Escuela Normal, Palma de Mallorca, Institut de Ciències de l'Educació-Universitat de les Illes Balears, I984; Gómez BARnusell, A.: El Magisterio como profesión. Estudio sociológico del magisterio nacional en las Islas Baleares, Barcelona, Editorial Ariel, 1972.

${ }_{21}$ Sobre la presencia de la música en los estudios de grado de Educación Infantil y Educación Primaria v. Díaz Mohedo, M. T.: «La Educación Musical en la Europa del futuro», Música y Educación: Revista Trimestral de Pedagogía Musical, 68 (2006), pp. 34-48.

${ }_{22}$ Berbel, N: «Anàlisi de l'evolució i situación actual de les escoles de música i/o dansa reconegudes a les Illes Balears», Investigació i Innovació Educativa i Socioeducativa, vol. 3, núm. 2 (20I2), pp. 36-66. 
APROXIMACIÓN HISTÓRICA Y METODOLÓGICA A LOS CURSOS DE PEDAGOGÍA MUSICAL DE LA UNIVERSITAT DE LES ILLES BALEARS (I977-I990)

LLORENÇ GELABERT GUAL

\section{Los cursos y el emergente movimiento coral en Mallorca}

El devenir de los cursos de música de la Universitat de les Illes Balears ha dependido ineludiblemente de los diferentes contextos educativos que se han ido sucediendo en España, del grado de reconocimiento de las enseñanzas musicales y de las instituciones encargadas de impartir dichas enseñanzas. Precisamente, estos aspectos han sido tratados en los dos puntos precedentes de este artículo. Sin embargo, el comienzo y origen de esta propuesta pedagógica debemos enmarcarlo en el ámbito de la música coral y de la demanda formativa que un grupo de directores llevó a cabo en Mallorca en la década de los setenta del siglo pasado.

El movimiento orfeonístico emerge en Mallorca a finales del siglo xIx. La visita del Orfeó Català i los Cors de Clavé, referentes del reconocido y ya consolidado movimiento coral catalán, reforzará sobremanera el ideal de culturización de la clase obrera. Se crean entonces en Mallorca un gran número de agrupaciones corales siguiendo ese modelo. La mayoría de ellas nacen vinculadas a sindicatos o ateneos culturales. Dicha vinculación a movimientos de reivindicación social y laboral enfatizará más la faceta de educar y culturizar a la gran masa social que el hecho de crear un producto musical de calidad. Por ello no se contemplan entonces conceptos como la pedagogía musical o la técnica de dirección musical ${ }^{23}$.

Con la llegada del régimen franquista, la idea de fomentar y acercar la formación musical a todas las capas sociales se ve de golpe truncada. Se inicia entonces un largo periodo de letargo formativo y cultural. Pervivirán, en cambio, los coros parroquiales y las agrupaciones musicales ligadas al ámbito religioso. Cabe destacar, en este ámbito, la gran tarea musical desempeñada por la Capella Clàssica del músico Joan M. Thomàs a lo largo de este periodo. Precisamente, el legado de esta agrupación marcará el camino a iniciativas posteriores ${ }^{24}$.

Finalizada la dictadura franquista se visualiza de nuevo un claro resurgimiento del movimiento musical y coral en Mallorca. En la década de los setenta se crean agrupaciones como el Orfeón Municipal de Palma, los Nins Cantors de Sant Francesc, la Coral del Tele-Club de Sineu, la Coral Polifònica de Bunyola o la Capella Mallorquina. Fruto de ese auge y del empuje asociacionista, empiezan a convocarse multitudinarios encuentros de agrupaciones corales. La creación de la Coral Universitària, con el amparo legal de la entonces Universitat de Palma de Mallorca, fortalecerá todavía más si cabe esa inercia, añadiendo el componente de excelencia musical ${ }^{25}$.

23 Sobre la música en Mallorca en el siglo xix v. Esteve, J. J.: La música a les Balears en el segle XIX, Palma de Mallorca, Documenta Balear, Quaderns d'Història Contemporània de les Balears, 56, 2007. En cuanto a la música coral en España en el xIX v. NAGORE, M.: «Historia de la música coral en España en el siglo XIX», en La música española en el siglo XIX, Oviedo, Servicio de Publicaciones de la Universidad de Oviedo, 1995, pp. 325-462.

${ }^{24}$ En cuanto a las agrupaciones musicales a lo largo del siglo xx v. Company, J.; Estelrich, P. y Moll, J.: Tres músics mallorquins: Antoni Torrandell, Joan Maria Thomàs i Antoni Matheu, Palma de Mallorca, Conselleria d'Educació i Cultura Govern Balear, Artistes de les Balears I, I985, pp. I09-I23.

${ }_{25}$ Sobre la Coral Universitat de les Illes Balears v. Company, J.: Coral Universitat de les Illes Balears. XXV anys. 1976-77/200I-2002, Palma de Mallorca, Universitat de les Illes Balears, 2003. 
APROXIMACIÓN HISTÓRICA Y METODOLÓGICA A LOS CURSOS DE PEDAGOGÍA MUSICAL DE LA UNIVERSITAT DE LES ILLES BALEARS (I977-I990)

LLORENÇ GELABERT GUAL

En septiembre del año 1973 se reúnen en el convento de Sant Francesc de Palma los directores musicales Francesc Batle (Antics Cantors de Sant Francesc), Baltasar Bibiloni (Massa Coral de Binissalem y Coral Tele-Club de Sineu), Jaume Conti (Coral Polifònica de Bunyola), Antoni Riera (Nins Cantors de Sant Francesc), Miquel Miró (Capella Oratoriana), Gori Marcús y Josep Vidal. En esta convocatoria se pone sobre la mesa el proyecto de crear una institución o federación que formalice el emergente movimiento coral, la creación de un espacio para el reciclaje y ampliación de conocimientos por parte de los mismos directores y la proyección a nuevos repertorios. En el año 1976 se constituye la junta gestora Corals de Mallorca. Diez años después se formaliza como Federació de Corals de Mallorca, con el objetivo de seguir promocionando y promoviendo el canto coral en Mallorca ${ }^{26}$.

Joan Company, entonces director de la Coral de Sant Joan, se convierte en el principal impulsor de la creación de un espacio de formación para directores y músicos en general. En el año 1975 asiste a los prestigiosos cursos de dirección coral de Cervera (Lleida). Establece allí contacto con Manuel Cabero ${ }^{27}$, una de las personalidades más destacadas del también emergente y vanguardista movimiento coral catalán. Este encuentro marcará el inicio de más de dos décadas de trabajo conjunto e ininterrumpido en los cursos que Baltasar Bibiloni y Joan Company crearán poco después en Mallorca. Concretamente, en el mes de agosto del año 1977 tuvo lugar en el Seminario Diocesano de Mallorca el I Curs de Música Coral. Contó con la presencia de más de cincuenta alumnos y se organizó bajo los auspicios de Corals de Mallorca y de la Escuela Universitaria de Formación del Profesorado de EgB de Palma. El primer profesor de dirección coral fue Manuel Cabero y Montserrat Pueyo se encargó de la técnica vocal ${ }^{28}$.

En Cataluña empezaba a consolidarse, desde principios de los setenta, un movimiento pedagógico en torno a la figura de Ireneu Segarra ${ }^{29}$, quien había creado un método de enseñanza musical enfocado a las escuelas de primaria. El contacto de Cabero con dicha corriente pedagógica facilitará la introducción de esta disciplina de forma optativa ya en el primer curso de Mallorca. Como primer

${ }_{26}$ Company, J.: «Cinquanta anys de la Capella Oratoriana. Una aproximació històrica al Cant Coral de Mallorca», en Capella Oratoriana, so anys de Música Coral (1947-1997), Palma de Mallorca, Capella Oratoriana, 1997, pp. I3-29.

${ }_{27}$ Manuel Cabero i Vernedas (1926-) fue el fundador del Cor Madrigal de Barcelona en el año I95I y su director musical durante cuatro décadas. Ha impartido clases de técnica de dirección coral en cursos de ámbito nacional e internacional. Recibió la Creu de Sant Jordi que otorga la Generalitat de Catalunya en el año 2002.

${ }_{28}$ En los programas de los cursos, desde la primera edición del año I977 hasta la última de I990, se puede consultar la oferta formativa, el profesorado presente en las distintas especialidades y otra información de interés.

29 Ireneu Segarra i Malla (1917-2005), compositor, director coral y pedagogo catalán. Fue el director musical de la Escolanía de Montserrat durante cuarenta y cinco años. Creó un método de enseñanza musical dirigido a la escuela primaria a partir de diferentes modelos vanguardistas ya experimentados en otros países de Europa. Su metodología fue concebida en base a las siguientes líneas de trabajo: la música como expresión de la vida y comunicación artística, la práctica musical, el canto como vehículo musical primordial, la presencia de la canción tradicional, trabajo del oído interno, estudio del lenguaje musical en toda su extensión, la audición, interpretación y estudio de obras, y la creación. 
APROXIMACIÓN HISTÓRICA Y METODOLÓGICA A LOS CURSOS DE PEDAGOGÍA MUSICAL DE LA UNIVERSITAT DE LES ILLES BALEARS (I977-I990) LLORENÇ GELABERT GUAL

representante de este movimiento pedagógico se cuenta entonces con la presencia de la profesora Maite Solà ${ }^{30}$. Desde ese momento el vínculo de los cursos con la pedagogía musical se consolida hasta el punto de crearse una especialidad en la cuarta edición. Baltasar Bibiloni, entonces profesor de música en la Escuela Normal de Magisterio, donde ya trabajaba en una línea cercana a la de Segarra, asume la jefatura de estudios dicha especialidad.

De alguna manera el colectivo de maestros de las Islas Baleares, sensibles a la formación musical, vio en la propuesta de Segarra el camino idóneo de cara a la introducción de esta disciplina en las aulas. Conscientes de que el nuevo marco legislativo abría las puertas a nuevas formas de enfocar dicha enseñanza, sólo faltaban las herramientas y recursos necesarios para llevarla a cabo. El método de Ireneu Segarra establecía precisamente una secuenciación de programaciones y actividades adaptadas a los distintos niveles de educación básica ${ }^{3 \mathrm{I}}$. A partir del vínculo que se estableció entre los organizadores de los cursos de Mallorca y la Escola de Pedagogia Musical-Mètode Ireneu Segarra, institución encargada de la aplicación y difusión del método en Cataluña ${ }^{32}$, se pudo contar entonces con la presencia de grandes pedagogos conocedores de primera mano de la metodología del músico catalán y de otros métodos ya aplicados exitosamente en otros países de Europa.

La oferta formativa de los cursos se incrementó edición tras edición. A las tres especialidades ya consolidadas de Pedagogía Musical, Dirección Coral y Técnica Vocal, se añadieron cursos de didáctica de la historia de la música, corrientes vanguardistas del siglo xx, iniciación a la tarea compositiva y talleres de ópera. Si bien es cierto que esta efervescencia de los cursos perduró durante más de una década, el nuevo contexto educativo que acontece en España a principios de la década de los noventa marcará un nuevo rumbo en esta oferta formativa.

En la edición del año I990, y coincidiendo con la introducción de la especialidad de Maestro en Educación Musical a la oferta de estudios de la Universitat de les Illes Balears del curso académico I990-9I, desaparece la especialidad de Pedagogía Musical de los cursos. El vacío formativo que cubrían hasta entonces pasa a manos ahora de la universidad. Ello comportó el reconocimiento académico de una formación restringida hasta ese momento al ámbito no formal o informal, precisamente. La especialidad fue sustituida por talleres de dirección orquestal y especialización en música del romanticismo, americana y de compositores nórdicos ${ }^{33}$.

30 Alrededor de la figura de Ireneu Segarra, y como espacio de difusión de su método, se creó a principios de los años setenta la Escola de Pedagogia Musical-Mètode Ireneu Segarra. Solà fue discípulo directo de Segarra y una profunda conocedora de su metodología, junto con otros pedagogos como Joan Casals o Santi Riera.

${ }_{31}$ En cuanto a la secuenciación del método de Ireneu Segarra y adaptaciones posteriores v. RiERA, S.: «L'aportació pedagògica», en Ireneu Segarra, mig segle de mestratge musical, Barcelona, Publicacions de l'Abadia de Montserrat, 1998, pp. 37-56.

${ }_{32}$ Sobre la Escola de Pedagogia Musical-Mètode Ireneu Segarra de Cataluña v. Cortina, J.: «L'Escola de Pedagogia Musical-Mètode Ireneu Segarra», en Ireneu Segarra, mig segle de mestratge musical, Barcelona, Publicacions de l'Abadia de Montserrat, 1998, pp. 57-62.

33 Cabe destacar que en las últimas ediciones se contó con la presencia de directores musicales de reconocido prestigio internacional como Pierre Cao, Anders Eby, Bruce Browne o José Antonio Sáinz Alfaro. Se crearon también seminarios dedicados a la interpretación operística, oratorio, lied y canción española. 
APROXIMACIÓN HISTÓRICA Y METODOLÓGICA A LOS CURSOS DE PEDAGOGÍA MUSICAL DE LA UNIVERSITAT DE LES ILLES BALEARS (I977-I990) LLORENÇ GELABERT GUAL

El resto de especialidades, con mayor o menor afluencia de alumnos, perduraron hasta la última edición del año 2006.

A través de la memoria del xvir Curso Internacional de Canto y Dirección Coral en las Islas Baleares, del año 1993, hemos tenido acceso a datos estadísticos de interés que nos dan una idea de la magnitud que alcanzaron los cursos en los años de mayor esplendor. Estos datos reflejan el periodo comprendido entre las ediciones de 1977 y 199i. Teniendo en cuenta que la especialidad de Pedagogía Musical, objeto de nuestro análisis, ya no se ofreció en la edición de I990, el lector puede hacerse una idea aproximada de la cantidad de alumnos que potencialmente la cursaron hasta su última edición ${ }^{34}$.

El número de alumnos matriculados y de profesores experimentó un considerable aumento con el paso de las ediciones. Una clara muestra de su eco y repercusión se manifiesta en la gran cantidad de alumnos procedentes de distintos puntos de la geografía española e incluso del extranjero. En el periodo 1977-1991 pasaron por los cursos un total de 716 alumnos con procedencia diversa:

- Mallorca: 453

- Menorca: I4

- Ibiza: 5

- Formentera: I

- Cataluña: I45

- Comunidad Valenciana: I2

- Madrid: 47

- Castilla y León: is

- Asturias: 7

- País Vasco: io

- Galicia: I

- Extremadura: 2

- Aragón: I

- Andalucía: I

- Castilla La Mancha: I

- Austria: I

El perfil de alumnos que asistía a los cursos era de lo más heterogéneo. Aunque en mayor número se trataba de perfiles relacionados con la docencia musical, encontramos también a personas procedentes de ámbitos que nada tenían que ver con la música y que veían en esta oferta formativa una posibilidad inédita de acceder a su aprendizaje. Encontramos a profesores de educación primaria (ЕGB), educación secundaria (BUP), conservatorios y escuelas de música, directores de corales infantiles, juveniles y adultos, cantantes, animadores de canto litúrgico, monitores de tiempo libre, musicólogos, pedagogos, psicólogos, religiosos, médicos, administrativos, economistas, farmacéuticos, arquitectos, abogados, diseñadores, ingenieros, químicos, peritos industriales, físicos, geólogos, etc.

34 Memoria del XVII Curso Internacional de Canto y Dirección Coral en las Islas Baleares, Aula de Música, ICE, Universitat de les Illes Balears, I993. 
APROXIMACIÓN HISTÓRICA Y METODOLÓGICA A LOS CURSOS DE PEDAGOGÍA MUSICAL DE LA UNIVERSITAT DE LES ILLES BALEARS (I977-I990) LLORENÇ GELABERT GUAL

La consolidación de los cursos se visualiza también en el alto grado de reincidencia por parte de la mayoría de alumnos asistentes. Casi un $80 \%$ de alumnos llegaba a asistir hasta a tres ediciones distintas, más de un $40 \%$ a cuatro, un $20 \%$ a cinco y casi un Io\% a seis. Ello deja de manifiesto la voluntad por parte de los organizadores y profesorado de ofrecer una formación continuada y de calidad.

Seguidamente aportamos toda una serie de datos e informaciones significativas de cada una de las ediciones, desde 1977 hasta la edición de i99o. Aparecen, en este orden, el nombre del curso (en la medida en que fueron añadiéndose o eliminando especialidades el título de los mismos fue cambiando), fechas de realización, lugar, especialidades (incluyendo seminarios y/o talleres), profesorado, dirección y coordinación, patrocinios y/o colaboraciones, así como otros comentarios que puedan resultar de interés:

I Curs de Música Coral: 31/07 al o6/o8 de 1977. Seminario Diocesano de Mallorca, Palma de Mallorca. Dirección coral y Técnica vocal/foniatría. Manuel Cabero (dirección coral), Montserrat Pueyo (técnica vocal y foniatría) y Maite Solà (pedagogía musical infantil). Joan Company (será director hasta la última edición del año 2005) y Baltasar Bibiloni (abandonará la dirección y coordinación de la especialidad de Pedagogía Musical en el año I99o coincidiendo con la desaparición de esta especialidad).

Desde la primera hasta la última edición de los cursos se interpretará un concierto de clausura, donde participarán los alumnos asistentes. La organización fomenta la idea de curso intensivo facilitando el alojamiento y manutención de los alumnos. Casi en la totalidad de los cursos el canto común y la técnica vocal serán materias comunes a todo el alumnado. La pedagogía musical ya se hace presente en esta primera edición, de la mano de la profesora catalana Maite Solà. No será hasta la tercera edición en que se reconozca como especialidad.

II Curs de Música Coral: 30/07 al 05/08 de 1978. Casa de espiritualidad Son Roca, Palma de Mallorca. Dirección Coral y Técnica vocal/foniatría. Manuel Cabero (dirección coral), Joan Cabero (técnica vocal), Montserrat Pueyo (técnica vocal i foniatría), Baltasar Bibiloni (pedagogía musical), Maite Solà (pedagogía musical infantil). Caixa de Pensions per a la Vellesa i d'Estalvis y Caixa de Pensions «la Caixa» de Catalunya i Balears.

III Curs de Música Coral a Mallorca: 05/08 al II/08 de I979. Casa de espiritualidad Son Roca, Palma de Mallorca. Pedagogía musical, Técnica vocal/foniatría y Didáctica de la historia de la música. Baltasar Bibiloni (pedagogía musical), Joan Cabero (técnica vocal), Manuel Cabero (dirección coral), Joan Company (didáctica historia de la música), Montserrat Pueyo (técnica vocal), Assumpta Valls (pedagogía musical). Caixa de Pensions per a la Vellesa i d’Estalvis y Caixa de Pensions «la Caixa» de Catalunya i Balears.

IV Curs de Música Coral i Pedagogia Musical a les Balears: 20/07 al 29/o7 de 1980. Colegio La Porciúncula, Palma de Mallorca. Iniciación a la lectura musical, Curso de introducción al método Ireneu Segarra, Dirección Coral A, Dirección Coral B y Técnica vocal. Baltasar Bibiloni (pedagogía musical), Joan Cabero (técnica vocal), Manuel Cabero (dirección coral), Joan Company (dirección coral), Francesc Crespí (pedagogía musical), Maria Teresa Malagarriga (pedagogía 
APROXIMACIÓN HISTÓRICA Y METODOLÓGICA A LOS CURSOS DE PEDAGOGÍA MUSICAL DE LA UNIVERSITAT DE LES ILLES BALEARS (I977-I990) LLORENÇ GELABERT GUAL

musical), Montserrat Pueyo (técnica vocal), Santi Riera (pedagogía musical), Montserrat Sobrevías (pedagogía musical). Caixa de Pensions per a la Vellesa i d'Estalvis, Caixa de Pensions «la Caixa» de Catalunya i Balears y Consell General Interinsular de la Conselleria d'Educació i Cultura.

V Curs de Música Coral i Pedagogia Musical a les Balears: 29/07 al 03/08 de 198r. Colegio La Porciúncula, Palma de Mallorca. Iniciación a la lectura musical, Introducción al método Ireneu Segarra, Método Ireneu Segarra (Parvulario I), Método Ireneu Segarra (EGB I), Método Ireneu Segarra (EGB 2), Dirección Coral I, Técnica Vocal i. Francesca Alomar (técnica vocal), Gaby Balthes (dirección coral), Margalida Barbal (pedagogía musical), Francesc Batle (pedagogía musical), Baltasar Bibiloni (pedagogía musical), Joan Cabero (técnica vocal), Manuel Cabero (dirección coral), Joan Company (dirección coral), Francesc Crespí (pedagogía musical), Montserrat Pueyo (técnica vocal), Santi Riera (pedagogía musical), Montserrat Sobrevías (pedagogía musical), Núria Quadrada (pedagogía musical), Ignasi Furió (pianista). Caixa de Pensions «la Caixa» de Catalunya i Balears (Obra Social Cultural) y Consell General Interinsular de la Conselleria d'Educació i Cultura.

VI Curs de Música Coral i Pedagogia Musical a les Balears: 17/07 al oi/o8 de 1982. Casa de espiritualidad Son Roca, Palma de Mallorca. Colegio La Porciúncula, Palma de Mallorca. Dirección Coral: Grupo A/Grupo B/Grupo C; Técnica Vocal: Grupo A/Grupo B/Grupo C; Pedagogía Musical Método Ireneu Segarra Música en Preescolar/Curso de introducción al método/Primer grado de parvulario/Primer grado de EGB/Segundo grado de EGB. Francesca Alomar (técnica vocal), Gaby Balthes (dirección coral), Margalida Barbal (pedagogía musical), Francesc Batle (pedagogía musical), Baltasar Bibiloni (pedagogía musical), Joan Cabero (técnica vocal), Manuel Cabero (dirección coral), Joan Company (dirección coral), Francesc Crespí (pedagogía musical), Montserrat Pueyo (técnica vocal), Santi Riera (pedagogía musical), Montserrat Sobrevías (pedagogía musical), Ignasi Furió (pianista). Caixa de Pensions «la Caixa» de Catalunya i Balears (Obra Social Cultural) y Consell Insular de Mallorca.

VII Curs de Música Coral i Pedagogia Musical a les Balears: 28/07 al 05/08 de 1983. Colegio La Porciúncula, Palma de Mallorca. Dirección Coral: Grupo A/Grupo B/Grupo C; Técnica Vocal: Grupo A/Grupo B/Grupo C; Pedagogía Musical Método Ireneu Segarra Música en Preescolar/Curso de introducción al método/ Primer grado de parvulario/Primer grado de EGB/Segundo grado de EGB/Música hasta $4 .^{\circ}$ curso de EGB; Coro piloto. Francesca Alomar (técnica vocal), Gaby Balthes (dirección coral), Francisco Alcalde (pedagogía musical), Baltasar Bibiloni (pedagogía musical), Joan Cabero (técnica vocal), Manuel Cabero (dirección coral), Joan Company (dirección coral), Francesc Crespí (pedagogía musical), Assumpta Malagarriga (pedagogía musical), Pere Noguera (expresión corporal), Josep Maria Pons (pedagogía musical), Montserrat Pueyo (técnica vocal), Santi Riera (pedagogía musical), Ignasi Furió (pianista). Caixa de Pensions «la Caixa» de Catalunya i Balears (Obra Social Cultural).

VIII Curs de Música Coral i Pedagogia Musical a les Balears: oI/o8 al Io/o8 de 1984. Colegio La Porciúncula, Palma de Mallorca. Dirección Coral: Grupo A/Grupo B/Grupo C; Técnica Vocal: Grupo A/Grupo B/Grupo C; Pedagogía Musical Método Ireneu Segarra Música en Preescolar/Curso de introducción al método/ 
APROXIMACIÓN HISTÓRICA Y METODOLÓGICA A LOS CURSOS DE PEDAGOGÍA MUSICAL DE LA UNIVERSITAT DE LES ILLES BALEARS (I977-I990) LLORENÇ GELABERT GUAL

Primer grado de parvulario/Primer grado de EGB/Segundo grado de EGB/Música hasta $4 .^{\circ}$ curso de EGB/Introducción a la Música del Siglo xx; Coro piloto. Francesca Alomar (técnica vocal), Gaby Balthes (dirección coral), Baltasar Bibiloni (pedagogía musical), Joan Cabero (técnica vocal), Manuel Cabero (dirección coral), Joan Company (dirección coral), Francesc Crespí (pedagogía musical), Joaquim Garrigosa (pedagogía musical), Carles Guinovart (Música s. Xx), Assumpta Malagarriga (pedagogía musical), Pere Noguera (expresión corporal), Josep Maria Pons (pedagogía musical), Montserrat Pueyo (técnica vocal), Elvira Querol (pedagogía musical), Ignasi Furió (pianista). Caixa de Pensions «la Caixa» de Catalunya i Balears (Obra Social Cultural) y Universidad de Palma de Mallorca.

IX Curs de Música Coral i Pedagogia Musical a les Balears: oI/o8 al o9/o8 de 1985. Colegio La Porciúncula, Palma de Mallorca. Dirección Coral: Grupo A/Grupo B/Grupo C; Técnica Vocal: Grupo A/Grupo B/Grupo C; Pedagogía Musical Método Ireneu Segarra Música en Preescolar/Curso de introducción al método/ Primer grado de parvulario/Primer grado de EGB/Segundo grado de EGB/Música hasta $4 .^{\circ}$ curso de EGB; Introducción a la Música del Siglo xx; Coro piloto. Francesca Alomar (técnica vocal), Gaby Balthes (dirección coral), Baltasar Bibiloni (pedagogía musical), Joan Cabero (técnica vocal), Manuel Cabero (dirección coral), Joan Company (dirección coral), Francesc Crespí (pedagogía musical), Joaquim Garrigosa (pedagogía musical), Carles Guinovart (música s. xx), Carmen Poch (pianista), Santi Riera (pedagogía musical), Josep Maria Pons (pedagogía musical), Montserrat Pueyo (técnica vocal), Elvira Querol (pedagogía musical), Ignasi Furió (pianista). Gori Marcús (será coordinador hasta la duodécima edición del año 1987. A partir de la siguiente edición la coordinación pasó a manos del Aula de Música de la Universidad de Palma de Mallorca). Federación de Corales de Mallorca, Caixa de Balears «Sa Nostra» y Aula de Música de la Universidad de Palma de Mallorca.

La entonces recién creada Federación de Corales de Mallorca concedió en esta edición ayudas para la inscripción a miembros de corales federadas. Estas ayudas se prolongaron hasta la vigésima cuarta edición de los cursos del año 2000.

$X$ Curs de Música Coral i Pedagogia Musical a les Balears: 31/o8 al o8/08 de 1986. Colegio La Porciúncula, Palma de Mallorca. Dirección Coral: Grupo A/Grupo B/ Grupo C; Técnica Vocal: Grupo A/Grupo B/Grupo C; Pedagogía Musical Método Ireneu Segarra Música en Preescolar/Curso de introducción al método/Primer grado de parvulario/Primer grado de EGB/Segundo grado de EGB/Música hasta $4 .{ }^{\circ}$ curso de EGB; Música del Siglo xx; Coro piloto. Francesca Alomar (técnica vocal), Gaby Balthes (dirección coral), Baltasar Bibiloni (pedagogía musical), Joan Cabero (técnica vocal), Manuel Cabero (dirección coral), Joan Company (dirección coral), Miquel Estelrich (pianista), Francesc Crespí (pedagogía musical), Nuria Lluveres (pedagogía musical), Carles Guinovart (música s. xx), Santi Riera (pedagogía musical), Josep Maria Pons (pedagogía musical), Montserrat Pueyo (técnica vocal), Elvira Querol (pedagogía musical), Ignasi Furió (pianista). Federación de Corales de Mallorca, Consell Insular de Mallorca y Aula de Música de la Universitat de les Illes Balears.

XI Curs de Direcció Coral, Tècnica Vocal i Pedagogia musical a les Balears: 30/07 al 07/08 de 1987. Colegio La Porciúncula, Palma de Mallorca. Dirección Coral: Grupo A/Grupo B/Grupo C; Técnica Vocal: Grupo A/Grupo B/Grupo C; Pedagogía Musical Método Ireneu Segarra Música en Preescolar/Curso de 
APROXIMACIÓN HISTÓRICA Y METODOLÓGICA A LOS CURSOS DE PEDAGOGÍA MUSICAL DE LA UNIVERSITAT DE LES ILLES BALEARS (I977-I990)

LLORENÇ GELABERT GUAL

introducción al método/Primer grado de parvulario/Primer grado de EGB/Segundo grado de EGB/Música hasta $4 .^{\circ}$ curso de EGB; Coro piloto. Francesca Alomar (técnica vocal), Gaby Balthes (dirección coral), Baltasar Bibiloni (pedagogía musical), Joan Cabero (técnica vocal), Manuel Cabero (dirección coral), Joan Company (dirección coral), Miquel Estelrich (pianista), Francesc Crespí (pedagogía musical), Eulàlia Salbanyà (técnica vocal), Núria Lluveres (pedagogía musical), Joaquim Miranda (pedagogía musical), Josep Maria Pons (pedagogía musical), Montserrat Pueyo (técnica vocal), Elvira Querol (pedagogía musical), Ignacsi Furió (pianista). Federación de Corales de Mallorca y Aula de Música de la Universitat de les Illes Balears.

XII Curs de Direcció Coral, Tècnica Vocal i Pedagogia musical a les Balears: 30/07 al o7/o8 de 1988. Colegio La Porciúncula, Palma de Mallorca. Dirección Coral: Grupo A/Grupo B/Grupo C; Técnica Vocal: Grupo A/Grupo B/Grupo C; Pedagogía Musical Método Ireneu Segarra Música en Preescolar/Curso de introducción al método/Primer grado de parvulario/Primer grado de EGB/Segundo grado de EGB/Música hasta $4 .^{\circ}$ curso de EGB/Tercer grado de EGB. Francesca Alomar (técnica vocal), Gaby Balthes (dirección coral), Baltasar Bibiloni (pedagogía musical), Joan Cabero (técnica vocal), Manuel Cabero (dirección coral), Joan Company (dirección coral), Miquel Estelrich (pianista), Francesc Crespí (pedagogía musical), Eulalia Salbanyà (técnica vocal), Núria Lluveres (pedagogía musical), Joaquim Miranda (pedagogía musical), Pere Noguera (escenificación), Josep Maria Pons (pedagogía musical), Montserrat Pueyo (técnica vocal), Jordi Ricard (técnica vocal), Elvira Querol (pedagogía musical), Miquel Àngel Segura (pianista), Ignasi Furió (pianista). Federación de Corales de Mallorca y Aula de Música de la Universitat de les Illes Balears.

Se introduce en esta edición, en la especialidad de Pedagogía Musical, la formación en el método adaptada al tercer grado de EGB.

XIII Curs de Direcció Coral, Tècnica Vocal i Pedagogia musical a les Balears: 29/07 al 07/08 de 1989. Colegio La Porciúncula, Palma de Mallorca. Dirección Coral: Grupo A/Grupo B/Grupo C; Técnica Vocal: Grupo A/Grupo B/Grupo C; Pedagogía Musical Curso de introducción al método Ireneu Segarra/Didáctica del solfeo. Francesca Alomar (técnica vocal), Gaby Balthes (dirección coral), Baltasar Bibiloni (pedagogía musical), Bartomeu Barceló (pedagogía musical), Joan Cabero (técnica vocal), Manuel Cabero (dirección coral), Joan Company (dirección coral), Miquel Estelrich (pianista), Francesc Crespí (pedagogía musical), Eulalia Salbanyà (técnica vocal), María M. Gómez (pedagogía musical), Santi Riera (pedagogía musical), Montserrat Pueyo (técnica vocal), Jordi Ricard (técnica vocal), Miquel Àngel Segura (pianista), Ignasi Furió (pianista), Tita Rotger (Yoga). Conselleria de Cultura, Educación y Deportes del Gobierno Balear y Federación de Corales de Mallorca.

Se introduce en esta edición un curso de Didáctica del Solfeo, dirigido a profesionales de la enseñanza del solfeo en conservatorios, escuelas de música, academias, preparadores de alumnos libres, etc. Se introducen por primera vez sesiones de yoga.

XIV Curs de Cant, Direcció Coral i Pedagogia Musical a les Balears: 28/o7 al 05/08 de 1990. Colegio La Porciúncula, Palma de Mallorca. Dirección Coral: Grupo A/Grupo B/Grupo C/Grupo D; Canto Grupo A/Grupo B/Grupo C; Taller de 
APROXIMACIÓN HISTÓRICA Y METODOLÓGICA A LOS CURSOS DE PEDAGOGÍA MUSICAL DE LA UNIVERSITAT DE LES ILLES BALEARS (I977-I990)

LLORENÇ GELABERT GUAL

ópera; Interpretación de la Música del Renacimiento; Curso de composición: estilos y grafías del siglo xx. Jordi Casas (dirección coral), Gaby Balthes (dirección coral), Manuel Cabero (dirección coral), Joan Company (dirección coral), Francesca Alomar (técnica vocal), Montserrat Pueyo (técnica vocal), Eulalia Salbanyà (técnica vocal), Enriqueta Tarrés (técnica vocal), Ignasi Furió (pianista), Margalida Furió (pianista), Miquel A. Segura (pianista), Roman Alís (composición). Conselleria de Cultura, Educación y Deportes del Gobierno Balear.

Se elimina la especialidad de Pedagogía Musical. Se introduce en su lugar el Taller de Ópera y el Curso de Composición. El comunicado literal que apareció en el programa del curso justificando dicha supresión decía lo siguiente: «La dirección del curso cree oportuno, dada la inmediata aparición de la figura de Diplomado en Educación Musical, impartir las disciplinas de Pedagogía Musical a lo largo del curso académico 90/9i, y no durante el curso de verano como era habitual. Oportunamente recibirá información sobre la realización de dos seminarios dedicados a la didáctica de otras tantas materias importantísimas dentro de la educación musical [...]». Cabe señalar que los seminarios de invierno no llegaron a realizarse.

\section{La pedagogía musical en los cursos}

«La música es patrimonio de todos los hombres y no propiedad exclusiva de una minoría. Debemos entonces hacerla accesible a todos. Y para ello no existe mejor fórmula que hacer música en la escuela» ${ }^{35}$. Esta y otras demandas efectuadas por Baltasar Bibiloni, coordinador de la especialidad de Pedagogía Musical en los cursos de música de la Universitat de les Illes Balears, nunca son proclamadas desde la gratuidad. Obedecían entonces a la finalidad de formar a un profesorado competente en música. Afirma en este sentido: «Urge en Mallorca un plan que posibilite a gente con ilusión, prepararse de forma eficaz. Toda iniciativa que estimule la formación de educadores para la música debería ser prioritaria si no se quiere mantener una cultura musical de fachada» ${ }^{36}$. En sus escritos son constantes las referencias tanto a las grandes figuras y pedagogos musicales del siglo xx como a los modelos educativos aplicados en otros países de Europa. En algunos de ellos la música aparece como una materia normalizada en la escuela, aportando un valor añadido al proceso educativo en general ${ }^{37}$.

En el caso de España, «venimos de una escuela sin música y por tanto nuestra sociedad, incluso en sus estratificaciones más elevadas, sufre de analfabetismo musical. Nadie ama lo que desconoce, he aquí la razón profunda de la impasibilidad con que la sociedad afronta el problema de la propia incultura musical» ${ }^{38}$. Con

35 Bibiloni, B.: «En el centenari del naixement de Zoltán Kodály», Diario de Mallorca, 23 de julio de 1982.

36 Bibiloni, B.: Música i educació bàsica. Lección inaugural del curso académico 1984-85 de la Escuela Universitaria de Formación del Profesorado de egB de Palma de Mallorca.

37 En cuanto a la presencia de la música en los modelos educativos de otros países v. CATEURA Mateu, M.: Por una educación musical en España. Estudio comparativo con otros países, Barcelona, Promociones y Publicaciones Universitarias, 1992.

38 Bibiloni, B.: op. cit. (32). 
APROXIMACIÓN HISTÓRICA Y METODOLÓGICA A LOS CURSOS DE PEDAGOGÍA MUSICAL DE LA UNIVERSITAT DE LES ILLES BALEARS (I977-I990)

LLORENÇ GELABERT GUAL

estas contundentes palabras Bibiloni define la situación de la enseñanza musical en España a mediados de la década de los setenta del siglo pasado. La implantación de la especialidad de Pedagogía Musical será el primer paso a favor de un cambio de tendencia y a la introducción de la música en las escuelas de las Islas Baleares. Las metodologías activas de Kodály, Orff o Dalcroze, ya aplicadas con éxito en otros países de Europa, se convertirán en la principal fuente de recursos de este proceso $^{39}$.

El nexo de colaboración de los cursos de Mallorca con la Escola de Pedagogia Musical-Mètode Ireneu Segarra de Cataluña abre la puerta a las metodologías activas anteriormente mencionadas ya desde el comienzo. El método de Ireneu Segarra, muy en consonancia con la idea del pedagogo húngaro Zoltán Kodály, gozaba ya de una gran aceptación en Cataluña. Su implantación en Mallorca a través de los cursos tendrá una repercusión similar. Gran parte de la comunidad de educadores musicales reconoce en nuestros días la importancia de la asimilación de esos nuevos conceptos y su legado vigente. Cabe destacar, en este sentido, el grado de aceptación e implantación de la metodología de Ireneu Segarra en los programas de formación musical de las diferentes etapas de la enseñanza obligatoria en los últimos años. Dicha influencia ha llegado también a las escuelas de música y a los conservatorios.

En cuanto a la didáctica aplicada del método Ireneu Segarra, se sigue una secuenciación de ocho niveles o cursos (divididos en cuatro ciclos de dos), adaptados a la Enseñanza General Básica entonces vigente y que venía contemplada en la LGE de 1970. En los cursos de Mallorca se impartieron los tres primeros ciclos equivalentes a los seis primeros cursos de Primaria, además de la previa e ineludible sensibilización musical dirigida a la etapa de Educación Infantil. Destaca el método por su adaptación a las diferentes instituciones o ámbitos de enseñanza musical: Educación Infantil, Primaria y Secundaria, escuelas de música y conservatorios. La primera edición del método, publicado como $E l$ meu llibre de música ${ }^{40}$ en los ocho niveles equivalentes a la enseñanza primaria de entonces, se ha visto complementada con más de cincuenta publicaciones, todas ellas dirigidas a su actualización y difusión. Entre estas destacan libros de texto, libro del alumno, libro del profesor, cancioneros, métodos para instrumento, materiales de audición y apoyo didáctico. Con anterioridad a esta primera edición del método se publicó el cancionero Juguem cantant ${ }^{41}$, que incluía cincuenta canciones creadas por Segarra y ordenadas de menor a mayor dificultad. Estas canciones fueran la herramienta básica de trabajo en los cursos de Mallorca, añadiendo posteriormente el repertorio de canciones tradicionales y populares de las Islas Baleares.

39 Sobre metodologías activas aplicadas a la música v. JonQuera, M. C.: «Métodos Históricos o Activos en Educación Musical», Revista Electrónica de LEEME (Lista Europea de Música en la Educación), I4 (2004). Recuperado de: http://musica.rediris.es/leeme/revista/jorquerao4.pdf.

$4^{\circ}$ Segarra, I.: El meu llibre de música, primer grau, Barcelona, Publicacions de l'Abadia de Montserrat, 1983.

${ }^{\mathrm{I}}$ SegarRA, I.: Juguem cantant. 50 cançons per a la iniciació musical, Barcelona, Publicacions de l'Abadia de Montserrat, 1973. 
APROXIMACIÓN HISTÓRICA Y METODOLÓGICA A LOS CURSOS DE PEDAGOGÍA MUSICAL DE LA UNIVERSITAT DE LES ILLES BALEARS (I977-I990) LLORENÇ GELABERT GUAL

Los cinco parámetros de acción musical a partir de los cuales se vertebran los distintos niveles que comprenden los cursos de música de la Universitat de les Illes Balears, en la especialidad de Pedagogía Musical, son los siguientes ${ }^{22}$ :

- Canción

- Formación vocal

- Formación del oído

- Ritmo-movimiento

- Audiciones

Los ejes metodológicos a partir de los cuales se trabajan los diferentes parámetros se basan en:

- Uso de la voz como instrumento principal

- Sensibilizar el sentido del oído

- Formar el sentido del ritmo a través del movimiento y la danza

- Establecer un contacto gradual pero directo con las grandes obras de la música universal

- Conocer el lenguaje musical básico: lectura y escritura

- Estimular la dimensión creativa a partir de la libre utilización de los elementos vividos, practicados y asimilados

La canción es el elemento y eje principal de aprendizaje de la música en la escuela. Se trabajará con un repertorio de canciones que abarque los distintos niveles de enseñanza musical que asume el maestro, desde los inicios de la Educación Infantil hasta el último curso de Educación Primaria. La elección de las canciones deberá contemplar el trabajo de aspectos rítmicos, melódicos, expresivos y/o teóricos, y se aprenderán siempre por imitación. Sólo al final del primer ciclo se iniciará el aprendizaje de canciones a través de la lectura de elementos rítmicos y melódicos sencillos, colectivamente y con la ayuda del maestro.

La educación de la voz, muy ligada a la canción, incide en aspectos de técnica vocal mediante ejercicios adaptados a los niños, a sus edades y tesituras vocales. Tiene especial importancia en este apartado el trabajo de la respiración y la posición corporal. Sin ser un trabajo demasiado prolongado, la técnica vocal debe estar siempre presente en las sesiones de clase.

La formación anditiva es un aspecto fundamental de cara a la formación musical integral de los niños. La fijación de las relaciones interválicas es el aspecto de maduración más lento dentro del proceso de formación, debido a su naturaleza abstracta. A menudo olvidada, una buena educación del oído se convierte en la sólida base de los futuros músicos. Sin esta no podemos hablar de educación musical completa. Cabe iniciarla ya en la etapa de Educación Infantil y mantenerla a

42 Estos parámetros de acción y las propuestas didácticas adjuntas a ellos aparecen en las programaciones de los cursos y anotaciones elaboradas por Baltasar Bibiloni y Francesc Crespí, ambos profesores en la especialidad de Pedagogía Musical. Dicha información se ha visto complementada con entrevistas personales llevadas a cabo con ambos profesores. En los párrafos siguientes aparecen de forma resumida los aspectos a trabajar en cada parámetro de enseñanza. 
APROXIMACIÓN HISTÓRICA Y METODOLÓGICA A LOS CURSOS DE PEDAGOGÍA MUSICAL DE LA UNIVERSITAT DE LES ILLES BALEARS (I977-I990)

LLORENÇ GELABERT GUAL

lo largo de toda la etapa de Educación Primaria. Un riguroso trabajo en este sentido implicará el reconocimiento de las distintas cualidades del sonido a muchos niveles por parte del niño.

Indisociable al trabajo del oído se incluirá paralelamente el trabajo sensorial del ritmo, ligado al movimiento. Se iniciará en edades muy tempranas, antes de la introducción al solfeo y deberá continuar durante todo el proceso de enseñanza musical. De los elementos que conforman la música, el ritmo es el más estrechamente relacionado con el movimiento corporal. Por ello nos serviremos didácticamente del movimiento en la clase de música. En estadios iniciales la educación motriz afecta a todos los niveles educativos: escritura, cálculo, música, etc. Abarca todos aquellos ejercicios que supongan una adecuación del niño al estímulo sonoro con el fin de lograr una regularidad y precisión en la percepción y exteriorización de las diferentes combinaciones rítmicas. A nivel estrictamente musical este parámetro trabaja aspectos tan importantes como la pulsación, las figuras rítmicas y el compás.

La audición es un complemento indispensable al resto de parámetros. Acerca las grandes obras de la literatura musical universal a los más pequeños formando su sensibilidad y criterio musical, fomentando a la vez la actitud de escucha activa. Contribuirá también a la introducción, descubrimiento y discriminación de elementos y conceptos musicales.

De cara a la asimilación e interiorización de estos elementos, el método apunta a un estudio por separado de los parámetros rítmicos, de la entonación y de la lectura. Utiliza el pentatonismo en el primer nivel de estudio de las relaciones melódicas, la fonomimia, el solfeo absoluto, la solmización y la práctica del dictado musical como herramientas de aprendizaje.

Todos y cada uno de los profesores que aparecen en las distintas ediciones de los cursos, además de trabajar en base a las directrices generales del método de Ireneu Segarra, destacaban por ser profundos conocedores del parámetro musical que impartían. En ningún caso se trataba de disciplinas inconexas que cada especialista aplicaba a partir de criterios propios. Baltasar Bibiloni, como coordinador de la especialidad, planificaba reuniones periódicas con el fin de organizar, secuenciar, debatir aspectos concretos y establecer líneas de trabajo común entre todo el profesorado.

Fueron profesores en la especialidad de Pedagogía Musical: Baltasar Bibiloni (lenguaje musical); Francesc Crespí (canción); Maite Solà, Elvira Querol y Josep M. Pons (educación infantil); Maria Teresa Malagarriga y Assumpta Malagarriga (educación sensorial); Santi Riera, Assumpta Valls, Núria Lluveres, Joaquim Miranda y Montserrat Sobrevias (método); Margalida Barbal (técnica vocal); Núria Quadrada y Pere Noguera (expresión corporal), y Joaquim Garrigosa (lenguaje musical conservatorios $)^{43}$.

El flujo e intercambio recíproco de profesorado que impartió docencia tanto en Mallorca como en Cataluña a lo largo de los años de existencia de la

43 Información extraída de los programas de los cursos, desde la primera edición del año 1977 hasta la desaparición de la especialidad de Pedagogía Musical, en la edición de 1990. Archivo personal de Joan Company. 
APROXIMACIÓN HISTÓRICA Y METODOLÓGICA A LOS CURSOS DE PEDAGOGÍA MUSICAL DE LA UNIVERSITAT DE LES ILLES BALEARS (I977-I990) LLORENÇ GELABERT GUAL

especialidad de Pedagogía Musical convirtió a los cursos en la extensión de la Escola de Pedagogia Musical-Mètode Ireneu Segarra y todo su legado metodológico en las Islas Baleares.

\section{Repercusión y legado}

Joan Company, director entonces del Aula de Música de l'Institut de Ciències de l'Educació (ICE) de la Universitat de les Illes Balears, cita textualmente en la memoria del XVII Curs Internacional de Cant i Direcció Coral a les Illes Balears: «Desde los inicios, los objetivos estuvieron bien definidos y geográficamente bien delimitados: estimular y fomentar una nueva situación musical en la sociedad de las Islas Baleares a partir de los medios musicales más elementales, la voz y la canción, y mediante nuevos planteamientos pedagógicos». A partir de esta premisa inicial y a lo largo de todas las ediciones, los cursos de música de la Universitat de les Illes Balears asumieron el rol formativo que las instituciones educativas de entonces no contemplaban. Desde el ámbito no formal abrieron camino hacia nuevas formas de entender la enseñanza de la música y su aplicación en las escuelas. El testigo lo han recogido la gran cantidad de maestros, profesores, músicos y directores que hoy, desde sus ámbitos y espacios de docencia en universidades, conservatorios, agrupaciones corales, escuelas de música, institutos o escuelas, siguen difundiendo su ideario pedagógico.

Este mismo documento, haciendo balance de las primeras quince ediciones, recoge: «Sin duda alguna, los cursos han incidido en el panorama musical de las Islas Baleares, sobre todo en el movimiento coral, en la enseñanza de la música en las escuelas y en el canto. Además han contribuido a la culminación de tres grandes objetivos: el resurgimiento del movimiento de corales infantiles en Mallorca, la creación de la Escola de Pedagogia Musical y la consolidación de la actual Federació de Corals de Mallorca».

¿Procede recuperar hoy en día un formato de cursos similar? La realidad nos describe un panorama incierto en cuanto a la formación de los maestros en música. En el nuevo marco de adaptación de los estudios universitarios al EEes desaparece la especialidad de Maestro en Educación Musical, junto al resto de especialidades que introdujo la LOGSE, a favor de una figura de maestro generalista con dos nuevas modalidades: Grado de Educación Infantil y Grado de Educación Primaria. La presencia de materias musicales en los nuevos planes de estudio, incluida la Mención de Música, no contempla ni una tercera parte de los contenidos de la antigua especialidad. La inexistencia, en las futuras generaciones de maestros, de un especialista en música nos retrotrae a la coyuntura que se vivía en la década de los setenta del siglo pasado. El debate está abierto. 